\title{
O Mundo Sombrio das Mulheres Vítimas de Violência: Uma Análise Qualitativa dos Boletins de Ocorrência.
}

\author{
Aline Pereira de Souza ${ }^{1}$, Maria José Sanches Marin ${ }^{1}$ e Paula Sales \\ Rodrigues $^{2}$ \\ ${ }^{1}$ Faculdade de Medicina Marília, Brasil | aline.psouza_enfer@hotmail.com; \\ marnadia@terra.com.br | https://orcid.org/0000-0003-4395-1482; https://orcid.org/0000- \\ 0001-6210-6941 \\ 2Universidade Estadual Paulista "Julio de Mesquita Filho" - UNESP - Câmpus de Botucatu, \\ Brasil | paulasalesrodrigues@gmail.com | https://orcid.org/0000-0002-8876-814X

\begin{abstract}
Resumo: O presente artigo visa identificar, verificar e apreciar os documentos da Delegacia da Mulher (DDM) da Polícia Judiciária do Município de Marília-SP na perspectiva de um estudo transversal na modalidade quantitativa por meio da técnica de análise temática, realizado a partir de boletins de ocorrência $(\mathrm{BO})$ de violência contra mulheres, com o intuito de revelar a magnitude da violência por uma abordagem qualitativa, contribuindo para o avanço na compreensão desse complexo fenômeno que carece de ações efetivas de saúde. A análise retratou fatores de vulnerabilidade às mulheres e as relações de desigualdade frente seus agressores, bem como os comportamentos coercitivos e de controle. As ameaças de morte ocorrem sob distintas condições e formas, as agressões físicas são degradantes, e ainda evidencia-se as agressões verbais, danos morais, intimidação, perseguição e injúrias. Violências que geram insegurança e medo nessas mulheres que buscam na ocorrência policial um direito que deveria ser irrevogável - a vida. Compreende-se, que é imprescindível a articulação de ações intersetoriais preventivas e de promoção em saúde com vistas ao
\end{abstract} \\ rompimento do ciclo da violência.
}

Palavras-chave: Violência Doméstica; Relações Familiares; Mulheres.

\section{The Dark World of Women Victims of Violence: A Qualitative Analysis of Police} Reports

\begin{abstract}
This article aims to identify, verify and examine the documents of the Women's Police Station (DDM) of the Judicial Police of Marilia city in the perspective of a cross-sectional study in the quantitative modality using the thematic analysis technique, carried out based on police reports of violence against women (BO), in order to reveal the magnitude of violence through a qualitative approach, contributing to the advancement in the understanding of this complex phenomenon that lacks effective health interventions. The analysis portrayed women vulnerability factors and relationships of inequality across their attackers, as well as the coercive behaviors and control. Death threats occur under different conditions and forms, physical assaults are degrading, and verbal assaults, moral damage, intimidation, persecution and injuries are also evident. Violence that generates insecurity and fear in women who seek in the police report a right that should be irrevocable life. It is understood that it is essential to articulate preventive intersectorial actions and promotion of health in order to break the violence cycle.
\end{abstract}

Keywords: Domestic violence; Family Relationships; Women.

\section{Introdução}

A violência é reconhecidamente um problema de saúde mundial e está relacionado com a construção da cidadania e da qualidade de vida da população, que pode e deve ser abordado pelos serviços de saúde por meio de estratégias de prevenção de doenças e agravos, promoção de saúde, assistência, recuperação e reabilitação. Destaca-se entre as formas de violência, aquelas proferidas contra pessoas consideradas em situação de maior vulnerabilidade como crianças, mulheres e idosos, as quais ocorrem predominantemente no âmbito familiar (Who, 2014). 
Há observações e estudos no campo de discussão acerca da violência contra a mulher, mencionadas como violência doméstica, violência intrafamiliar, violência doméstica e familiar, violência doméstica e sexual, violência de gênero, e/ou violência por parceiro íntimo. No âmbito jurídico, o termo comumente adotado é de violência doméstica contra a mulher, devido a lei Maria da Penha - Lei n. 11340 (Brasil, 2006), a qual cria mecanismos para coibir e prevenir a violência doméstica contra a mulher. No presente artigo manteremos o termo violência doméstica considerando os aspectos relacionados aos núcleos de cuidado em saúde: a família e o domicílio.

A violência contra a mulher é de tal magnitude que se postula, internacionalmente, como problemática da Saúde Pública. A perpetuação de tal violência doméstica apesar dos avanços institucionais, políticos e jurídicos dá-se por dificuldades relacionadas à impunidade, ineficácia prática em intervenções e prevenção, bem como a ineficiência de políticas públicas (Billand \& Paiva, 2017).

Segundo D'Oliveira e Schraiber (2013) mulheres em situação de violência buscam alternativas para superar a situação, sendo ativas no sentido de buscar interromper a violência. O pedido de ajuda à rede informal, formada por família e amigos é mais frequente do que à rede formal. As razões relatadas por essa busca foram impulsionadas pelo medo, dor, sofrimento e desejo de acabar com a situação. Quanto mais grave a violência, maior a procura por apoio, o que faz com que os casos que acorrem aos serviços especializados sejam já bastante graves.

Pesquisa realizada pela Organização Panamericana de Saúde (Opas, 2011) replicada no Brasil (Meneghel, et al, 2011) demonstra como a busca das mulheres afetadas pela violência doméstica pode ser difícil e cheia de obstáculos. Informações inadequadas e atitudes não acolhedoras e crivadas de julgamento moral são frequentes nos percursos relatados nas investigações.

Estima-se que cerca de quarenta mil brasileiras buscam o Sistema Único de Saúde (SUS) para tratamentos devido à violência, representando um custo anual superior a cinco milhões de reais. Todavia, esse valor não representa o gasto real do setor saúde, visto que se limita aos registros de internações associados ao agravo, não contemplando a totalidade das demandas dessa população, como as procuras na Atenção Básica, as repercussões familiares envolvendo filhos e a própria conjugalidade (Martins et al, 2018).

Estudos alertam que presenciar a violência entre os pais relaciona-se ao comprometimento no rendimento escolar e a vulnerabilidade para o uso de drogas. Além disso, pessoas com histórico de abusos familiares na infância e/ou adolescência tendem a reproduzi-lo nas relações interpessoais, favorecendo a intergeracionalidade da violência doméstica (Martins et al, 2018; Wagner et al, 2019).

Frente a discussão acima, a presente análise visa revelar a magnitude da abordagem qualitativa dos boletins de ocorrência, o que contribui para avanços na compreensão desse complexo fenômeno que carece de ações efetivas de saúde.

\section{Objetivo}

Analisar os motivos das ocorrências policiais de violência contra a mulher.

\section{Metodologia}

Trata-se de um estudo transversal na modalidade quantitativa por meio da técnica de análise temática, realizado a partir de boletins de ocorrência $(\mathrm{BO})$ de violência contra mulheres. Foi realizada em um Município de médio porte do interior de São Paulo, com uma população de 216.745 habitantes (IBGE, 2012) e teve como cenário para a coleta de dados a Central de Polícia Judiciária da Polícia Civil do Estado de São Paulo do município, mais especificamente na Delegacia de Defesa da Mulher. 
Foi desenvolvida por meio de análise documental que consiste em identificar, verificar e apreciar os documentos que permite uma contextualização das informações com o intuito de extrair um reflexo objetivo de uma fonte original (Souza, Kantorski, Luis, 2011). Tratase de uma forma de coleta e análise de dados com alto potencial informativo e consiste em uma série de operações que visam estudar documentos no intuito de compreender as circunstâncias e elucidar os conteúdos expressos (Souza, J. et al; 2017).

Foram incluídos no estudo os (B.O) realizados na referida delegacia, no mês de Janeiro de 2020, seguindo-se um roteiro de coleta de dados referentes as características sociodemográficas da mulher (idade, estado civil, escolaridade, cor da pele e ocupação profissional) e tipo de violência.

As fichas de registro das ocorrências foram disponibilizadas aos pesquisadores em uma sala localizada na própria sede da delegacia. Foram analisadas 50 ocorrências, a partir das 147 existentes, as quais foram selecionadas de acordo com a ordem de digitação do total de fichas, sendo que a coleta foi encerrada no momento que se observou a saturação dos dados.

A saturação significa o momento da pesquisa no qual a coleta de novos dados não traria mais esclarecimentos para o objeto estudado. Assim, o tamanho da amostra, é resultante da heterogeneidade da população que será pesquisada e preza-se pela quantidade e qualidade de dados (Minayo, C; 2017).

Para a análise dos dados obtidos, optou-se pela técnica da Análise Temática (AT) que é um método analítico qualitativo que busca identificar, analisar e relatar padrões (tema) dentro dos dados e interpretar vários aspectos do tema de pesquisa (Virginia Braun \& Victoria Clarke; 2006).

O processo de codificação dos dados não é fixado a priori, pois os temas são extraídos dos próprios dados, o que permite grande flexibilidade e colabora para uma análise interpretativa sobre os dados. Assim como as demais formas de análise de dados qualitativos a AT também preza pela busca de padrões, flexibilidade, homogeneidade interna dos temas e heterogeneidade externa entre eles (Souza, Kantorski, Luis, 2019).

Para as autoras, um tema capta algo importante que é abstraido dos dados em relação à pergunta de pesquisa e representa um significado padronizado a partir do conunto de dados obtidos, podendo ou não aparecer com grande prevalência no conjunto das informações (Virginia Braun \& Victoria Clarke; 2006).

Na operacionalização da análise temática, procura-se padrões de significados e questões relevantes nos dados, sendo que a elaboração dos temas ocorre por meio de sucessivas aproximações com os dados.

As autoras apresentam a análise temática em seis fases, entretanto, admitem que não se trata de um processo linear, sendo necessário ser flexibilidade e exaustivo na interação com os dados, em movimentos de "idas e vindas" para que os temas contemplem percpções ricas e complexas.

Descrevem-se a seguir as fases a serem seguidas: 1. familiaridade com os dados, que compreende a imersão por meio de leituras repetidas dos dados de forma a se aproximar da profundidade e amplitude do conteúdo.

Recomenda-se que o proprio pesquisador faça a coleta e a transcrição dos dados caso sejam obtidos por meio de entrevistas ou discursos, visando a familiarização com o conteúde de forma ampla. Leituras repetidas e ativa são necessárias para a busca de padrões e significados. Durante essa fase recomenda-se registrar as principais idéias para a codificação subsequente. 2. a produção de codigos iniciais, esta fase segnifica iniciar a produção de códigos, partir dos dados.

Os códigos representam um conteúdo semantico ou latente que se referem ao segmento ou elemento mais básico dos dados brutos. Difere-se, portanto, das unidades de análise ou temas, pois estes, muitas vezes, são mais amplos. 
Nesta fase, trabalha-se sistematicamente com o conjunto dos dados que foram obtidos, buscando identificar aspectos interessantes e significativos do texto. Deve ser codificado do banco de dados. 3. procura por temas - trata-se da fase que o foco da análise e ajustado para temas mais abrangente.

Envolve a triagem dos diferentes códigos em temas potenciais, por meio de agrupamentos dos extratos relevantes aos temas que estão sendo construídos.

Recomenda-se que nesta fase sejam utilizadas representações visuais para auxiliar na classificação dos diferentes códigos em temas.

$\mathrm{Na}$ finalização terá uma coleção de temas e subtemas. É nesta fase que começam a surgir as relações. Alguns códigos podem se transformar em temas, outros em subtemas e alguns podem até desaparecer. 4. revisitar os temas - esta fase envolve essencialmente o refinamento dos temas, leva em consideração os critérios de homogeneidade interna e heterogeneidade externa, sendo necessário, muitas vezes, retomar a codificação dos dados até que se consiga criar um mapa temático satisfatório. 5. Definir e nomear os temas, ou seja, é identificada a essência do assunto de cada tema.

Recomenda-se que os extratos não sejam para fraseados, mas que seja identificado o que é interessante sobre eles e porquê.

Como parte do refinamento é identificado se um tema tem ou não subtemas, podendo haver também hierarquia de sentidos dentro dos dados. Um determinado tema não deve dar conta de muitos aspectos ou ser muito complexo e diversificado. 6. Produção do relatório - tem início quando o conjunto de tema já foram totalmente trabalhados e iniciase a análise final e a escrita do relatório.

Neste relatório os extratos das falas dos participantes precisam ser incorporados à narrativa analítica, visando ilustrar o conteúdo que se pretende mostrar (Virginia Braun \& Victoria Clarke; 2006).

O estudo contou com a aprovação do Comitê de Ética e Pesquisa com Seres Humanos da instituição proponente sob parecer n.4.265.994 e CAAE: 36628920.8.0000.5413 Considerando que se trata de um estudo de análise documental, foi solicitada a dispensa do Termo de Consentimento Livre e Esclarecido. Os BO analisados receberam numeração em ordem crescente B1 a B50.

\section{Resultados}

\subsection{Caracterização das Vítimas}

Em relação a caracterização das vítimas, identifica-se que a violência perpassa de forma significativa tanto para as mulheres na faixa etária jovem-adulto quanto para as adultas mais velhas.

Evidencia-se, embora de forma pouco expressiva, maior incidência em mulheres mais velhas. Quanto ao estado civil, verifica-se que a maioria das vítimas são casadas ou constituídas em união estável. Expressa-se, também de forma relevante, as mulheres solteiras. No que tange a escolaridade, a maioria possui o ensino médio e encontram-se empregadas.

Revela-se que em sua maioria, as vítimas se auto declaram brancas e pardas. Considerando os tipos de violências, constata-se que a violência psicológica é a que mais acomete as vítimas, seguida da violência física; da moral e a financeira.

Ressalta-se ainda, que há vítimas que sofrem mais de um tipo de violência. 
Tabela 1. Características sociodemográficas das vítimas $\mathrm{N}=(50)$.

\begin{tabular}{|c|c|c|}
\hline & & Mulheres Vítimas \\
\hline \multirow[t]{2}{*}{ Idade } & 18 a 30 anos & $22(44 \%)$ \\
\hline & 31 a 59 anos & $28(56 \%)$ \\
\hline \multirow[t]{4}{*}{ Estado Civil } & Solteira & $20(40 \%)$ \\
\hline & Casada ou União Estável & $22(44 \%)$ \\
\hline & Divorciada & 06 (12\%) \\
\hline & Sem Informação & $02(4 \%)$ \\
\hline \multirow[t]{4}{*}{ Escolaridade } & Ensino Fundamental & 05 (10\%) \\
\hline & Ensino Médio & $12(24 \%)$ \\
\hline & Ensino Superior & 05 (10\%) \\
\hline & Não Informou & $28(56 \%)$ \\
\hline \multirow[t]{4}{*}{$\begin{array}{l}\text { Ocupação } \\
\text { Profissional }\end{array}$} & Empregada & $30(60 \%)$ \\
\hline & Desempregada & $06(12 \%)$ \\
\hline & Estudante & $03(6 \%)$ \\
\hline & Não Informado & $11(22 \%)$ \\
\hline \multirow[t]{3}{*}{ Cor da Pele } & Branca & $30(60 \%)$ \\
\hline & Negra & 05 (10\%) \\
\hline & Parda & $15(30 \%)$ \\
\hline \multirow[t]{4}{*}{ Tipo de violência } & Física & $22(44 \%)$ \\
\hline & Psicológica & $32(64 \%)$ \\
\hline & Moral & 08 (16\%) \\
\hline & $\begin{array}{l}\text { Financeira } \\
\text { Mais de um tipo de Violência }\end{array}$ & $\begin{array}{c}03(6 \%) \\
15(30 \%)\end{array}$ \\
\hline
\end{tabular}

\subsection{Temas e Subtemas}

Tabela 2. Apresentação dos Temas e Subtemas.

\begin{tabular}{cl}
\hline Temas & \multicolumn{1}{c}{ Subtemas } \\
\hline Ameaça de morte & Sob efeito de álcool e drogas E7, E10 \\
& Para ela e familiares. E2 \\
& Após recusa de reatar o relacionamento/Não aceita separação. E1, E9 \\
& Alegando ter posse de uma arma calibre 22. E8 \\
& Alegando que irá disparar uma arma na sua cabeça e dos familiares. E22 \\
& Dizendo que irá" rasgar com faca" E23 \\
& Exigindo dinheiro. E8 \\
& Alegando ser do PCC E40 \\
& Dizendo que será com tiro e que vai quebrar as pernas e arrancar a cabeça. \\
& E44 \\
No local de trabalho E5 & Com faca. E41, E43, E44, E45 \\
Mesmo com medida protetiva 3 & Por ter encontrado drogas ilícitas na carteira. E17
\end{tabular}




\begin{tabular}{|c|c|}
\hline Temas & Subtemas \\
\hline Agressão física & $\begin{array}{l}\text { Chute. E11 } \\
\text { Empurrão. E14, E21 } \\
\text { Colocou contra a parede e apertou o pescoço. E9 } \\
\text { Cabeçada E14 } \\
\text { Espancamento com lesões em olho e boca E10 } \\
\text { Socos e mordidas E19 e E49 } \\
\text { Puxões de cabelo E12, E20 } \\
\text { Tapas E 25, E28 } \\
\text { Socos, cadeiradas. E31 } \\
\text { Socos na face E34 } \\
\text { Espancamento. E33 } \\
\text { Sem especificação. E35, E15 } \\
\text { Tentativa de sufocação E48, E49 }\end{array}$ \\
\hline Danificou materiais & $\begin{array}{l}\text { após uso de BA e passou danificar móveis da casa. E32 } \\
\text { Danificou celular E30, E37 } \\
\text { Roubo de dinheiro e notebook E42 }\end{array}$ \\
\hline Ofensa verbal & $\begin{array}{l}\text { "você vai ver o que vai te acontecer, você vai se ferrar, vai pagar" E30 } \\
\text { Ameaçou de colocar fogo na casa } 32 \\
\text { Marido sempre a xingou, é controlador e possessivo. E6 } \\
\text { Ofensa de teor preconceituoso. E50 } \\
\text { Acusa de ter causado a separação. E47 } \\
\text { Proferiu palavras de baixo calão. E38, E39, E45 }\end{array}$ \\
\hline $\begin{array}{l}\text { Importunação/perseguição } \\
\text { e intimidação/injúria. }\end{array}$ & $\begin{array}{l}\text { Após separação foi importuná-la no emprego. E26, } \\
\text { Perseguindo, intimidando e dizendo que não a deixará em paz } \\
\text { Recebeu mensagem com tom ameaçador. E16 } \\
\text { Comportamento agressivo por ter encontrado drogas ilícitas na carteira. E17 } \\
\text { Após separação companheiro comparece na frente de casa. E24 }\end{array}$ \\
\hline
\end{tabular}

\section{Discussão}

Embora na literatura sejam encontrados estudos que exploram os $\mathrm{BO}$ de violência contra a mulher, na maioria deles, os dados são apresentados quantitativamente. No presente estudo foi realizada uma abordagem qualitativa, o que contribui para o avanço na compreensão desse complexo fenômeno; entretanto, as limitações são reconhecidas uma vez que a descrição existente nesses registros não explora a profundidade e o sentido da ocorrência.

Já os temas levantados revelam os trajetos em busca de ajuda que vão desde o silêncio até o grito de socorro. Caminhos que passam por processos pessoais e sociais de cada mulher, regido por intensos sentimentos, como a vergonha e o medo, para se chegar às declarações acima abstraídas. Portanto, é imprescindível considerar as expressões progressivas e cíclicas da violência doméstica bem como suas repercussões para mulheres e filhos, nos espaços jurídico-policiais, sociais e/ou na saúde (Lettiere \& Nakano, 2001; Lucena et al, 2016).

Os estudos identificam e associam alguns fatores da vida da mulher a uma condição de vulnerabilidade à violência, dentre esses encontra-se as variáveis socioeconômicas; o não esclarecimento ou reconhecimento sobre a violência associado a baixa escolaridade e a baixa autonomia, seja financeira ou de processos decisórios da vida. Já a propensão ao álcool e ao tabaco têm sido considerada tanto como fatores de vulnerabilidade quanto de escape aos traumas. Todos esses levam a baixa autoestima, vergonha, sentimento de culpa e estigmatização reforçando o ciclo da violência (Leite et al, 2017; Dos Santos et al, 2020; Crus \& Irff, 2019).

Diferenciar as ameaças de morte como categoria de análise traduz a gravidade dessas violências, fato que deveria ser reconhecido e interpretado pelos profissionais dos serviços da rede de enfrentamento à violência a partir de seu contexto e construção sociocultural reforçando a segurança das mulheres e garantindo um direito fundamental - a vida (Vieira e Hasse, 2016). 
Características pessoais do autor da violência, como o consumo de álcool masculino e aspectos do relacionamento afetivo foram identificados como disparadores das agressões entre parceiros, descrito por Bhona et al (2020). Não há uma homogeneidade da violência psicológica ou ameaça à vida, mas a característica predominante do agressor está no controle coercitivo ou comportamentos de controle. Identificar os padrões de controle, como abuso econômico e/ou emocional, uso de crianças, ameaças e intimidação, monitoramento constante, culpabilização da vítima, invocação do privilégio masculino e ameaças que envolvam o trabalho ou família do parceiro, possibilitaria atuações efetivas na prevenção de tais violências e seus agravos.

A literatura também retrata fatores que precedem o feminicídio, tais como residir em área rural, sofrer violência física e reincidência de violência, sendo este último o maior dentre os demais fatores (Straus \& Gozjolko 2014; Johnson, 2011; Barros, 2018).

A agressão física referida na análise, reforça os achados bibliográficos dessa tipificação. O rosto é a parte mais visível do corpo e contribui para a autoimagem do indivíduo. Os traumas maxilofaciais estão comumente associados a essas agressões em diferentes graus de danos representando um desafio para os serviços de saúde desde seu reconhecimento (Garcez, 2019).

As repercussões na vida da família são imensuráveis, pois refletem nas relações familiares posteriores, sendo que a qualidade do funcionamento familiar influencia significativamente no bem-estar e na saúde das pessoas, perpassando por todas as etapas do ciclo da vida. Há estudos que retratam que as crianças que vivenciaram situações de violência entre os pais tinham maior possibilidade de repetir o ato de violência quando adulto, indicou que os homens que testemunharam violência eram mais propensos a perpetrar a mesma, sugerindo uma transmissão intergeracional da violência. (Reisig \& Holtfreter, 2018; Islam et al, 2017).

A invisibilidade da violência ainda é um desafio. É necessário o reconhecimento da humanização dos atendimentos, melhorar a infraestrutura, criar serviços especializados, principalmente àqueles previstos pela Lei Maria da Penha (Brasil, 2020).

Apesar do avanço representado pelas iniciativas legislativas, de políticas públicas e de organização, as muitas dificuldades ainda persistem. Os sistemas de saúde têm papel crucial em resposta intersetorial à violência contra as mulheres. (Meneghel et al, 2011; Lucena et al, 2016; Moreno et al, 2015).

\section{Conclusão}

A partir dessa análise pode-se confirmar que se trata de uma relação de extrema desigualdade, decorrente de fatores que colocam a mulher em situação de vulnerabilidade frente ao seu agressor. Compreende-se, portanto, que é imprescindível ações mais efetivas, especialmente em relação às medidas preventivas e de promoção em saúde, com vistas ao rompimento do ciclo da violência.

Foi possível constatar que as ameaças de morte ocorrem sob distintas condições e formas, gerando insegurança e medo entre essas mulheres e embora tenham a obtenção da medida protetiva, nem sempre a mesma é respeitada e cumprida. As agressões físicas ocorrem de forma degradante, bem como os danos materiais, as agressões verbais e outras formas de violência como a intimidação, perseguição e injúria.

Os recursos legais da atualidade, como a Lei Maria da Penha, favorecem o rompimento do ciclo da violência, entretanto, ainda há um longo percurso para ações intersetoriais que promovam integralidade, cuidado, e autonomia das mulheres. 


\section{Referências}

Who. Global status report on violence prevention 2014. Geneva: World Health Organization; 2014.

Lucena, K. D. T. et al. Analysis of the cycle of domestic violence against women. Journal of Human Growth and Development, São Paulo, v. 26, n. 2, p. 139-146, 2016.

Michael D. Reisig \& Kristy Holtfreter (2018): The victim-offender overlap in lateadulthood, Journal of Elder Abuse \& Neglect, DOI: 10.1080/08946566.2018.1426512 To link to this article: https://doi.org/10.1080/08946566.2018.1426512

D'Oliveira AFPL, Schraiber LB. Mulheres em situação de violência: entre rotas críticas e redes intersetoriais de atenção / Violence against women: between critical path and assistance multisectoral networks. Rev Med (São Paulo). 2013 abr.-jun.;92(2):134-40

Leite FMC, Amorim MHC, Wehrmeister FC, Gigante DP. Violence against women, Espirito Santo, Brazil. Rev Saude Publica 2017; 51:33.

dos Santos, I. B., Leite, F. M. C., Amorim, M. H. C., Maciel, P. M. A., \& Gigante, D. P. (2020). Violence against women in life: study among Primary Care users. Ciência \& Saúde Coletiva, 25(5), 1935+. https://link.gale.com/apps/doc/A629396481/AONE?u=capes\&sid=AONE\&xid=db6febb4

Cruz, M. S., \& Irffi, G. (2019). What is the effect of violence against Brazilian women on their selfperception of health? /Qual o efeito da violencia contra a mulher brasileira na autopercepcao da saude? Ciência \& Saúde Coletiva, 24(7), 2531+. https://link.gale.com/apps/doc/A598424341/AONE?u=capes\&sid=AONE\&xid=d87375f3

Garcez, Ramiro Heleno Mesquita, et al. "Characterization of oral maxillofacial lesions resulting from physical aggression: differences between genders/Caracterizacao de lesoes bucomaxilofaciais decorrentes de agressao fisica: diferencas entre genero." Ciência \& Saúde Coletiva, vol. 24, no. 3, 2019, p. 1143+. Gale Academic OneFile, link.gale.com/apps/doc/A583693806/AONE?u=capes\&sid=AONE\&xid=9dc8b65f. Accessed 5 Mar. 2021.

Islam MJ, Rahman M, Broidy L, Haque SE, Saw YM, Duc NH, Haque MN, Rahman MM, Islam MR, Mostofa MG. Assessing the link between witnessing inter-parental violence and the perpetration of intimate partner violence in Bangladesh. BMC Public Health. 2017 Feb 10;17(1):183. doi: 10.1186/s12889-017-4067-4. PMID: 28187721; PMCID: PMC5303211.

Brasil. Lei no. 11.340, de 07 de agosto de 2006. Cria mecanismos para coibir a violência doméstica e familiar contra a mulher, altera o Código de Processo Penal, o Código Penal e a Lei de Execução Penal; e da outras providências. Diário Oficial da União 2006. Brasília, DF

Brasil. Lei no 13.984, de 3 de abril de 2020. Altera o art. 22 da Lei oㅜ 11.340, de 7 de agosto de 2006 (Lei Maria da Penha), para estabelecer como medidas protetivas de urgência frequência do agressor a centro de educação e de reabilitação e acompanhamento psicossocial. Diário Oficial da União: seção 1, Brasília, DF, ano 157, n. 117, p. 4, 3 abr. 2020.

Martins, L. C. A. et al. Violência de gênero: conhecimento e conduta dos profissionais da estratégia saúde da família. Revista Gaúcha de Enfermagem, Porto Alegre, v. 39, p. 1-10, 2018.

Junior, E. G. (2017). Análise documental: uma metodologia da pesquisa para a Ciência da Informação (07th ed.).

Souza, J. et al. "Análise documental e observação participante na pesquisa em saúde mental documentary." (2011).

Minayo, C. (2017). Amostragem e Saturação em Pesquisa Qualitativa: consensos e controvérsias.

Virginia Braun \& Victoria Clarke (2006): Using thematic analysis in psychology, Qualitative Research in Psychology, 3:2, 77-101

Souza, Luciana Karine de. (2019). Pesquisa com análise qualitativa de dados: conhecendo a Análise Temática. Arquivos Brasileiros de Psicologia, 71(2), 5167. https://dx.doi.org/10.36482/1809-5267.ARBP2019v71i2p.51-67 
Meneghel SN, Bairros F, Mueller B, Monteiro R, Oliveira LP, Collaziol ME. Rotas críticas de mulheres em situação de violência: depoimentos de mulheres e operadores em Porto Alegre, Rio Grande do Sul, Brasil. Cad Saúde Pública. 2011;27:743-52. http://dx.doi.org/10.1590/S0102-311X2011000400013

Billand, J.; PAIVA, V. S. F. Desconstruindo expectativas de gênero a partir de uma posição minoritária: como dialogar com homens autores de violência contra mulheres?. Ciência \& Saúde Coletiva, Rio de Janeiro, v. 22, n. 9, p. 2979-2988, 2017.

D'Oliveira, A. F. P. L.; SCHRAIBER, L. B. Mulheres em situação de violência: entre rotas críticas e redes intersetoriais de atenção. Revista de Medicina, São Paulo, v. 92, n.2, p. 134-140, 2013.

Wagner, J., Jones, S., Tsaroucha, A., and Cumbers, H. ( 2019) Intergenerational Transmission of Domestic Violence: Practitioners' Perceptions and Experiences of Working with Adult Victims and Perpetrators in the UK. Child Abuse Rev., 28: 39- 51. https://doi.org/10.1002/car.2541.

Vieira, E. M., \& Hasse, M. (2016). Percepções dos profissionais de uma rede intersetorial sobre o atendimento a mulheres em situação de violência. Interface - Comunicação, Saúde, Educação, 21(60), 52-62. doi:10.1590/1807-57622015.0357 García-Moreno C, Hegarty K, d'Oliveira AF, Koziol-McLain J, Colombini M, Feder G. The health-systems response to violence against women. Lancet. 2015 Apr 18;385(9977):1567-79. doi: 10.1016/S01406736(14)61837-7. Epub 2014 Nov 21. Erratum in: Lancet. 2015 Apr 18;385(9977):1510. PMID: 25467583.

Bhona, Fernanda Monteiro de Castro et al . Intimate Partner Violence: Controlling Behavior and Triggers of Aggression. Paidéia (Ribeirão Preto), Ribeirão Preto , v. 30, e3032, 2020 Available from <http://www.scielo.br/scielo.php?script=sci_arttext\&pid=S0103863X2020000100204\&Ing=en\&nrm=iso >. access on 04 Mar. 2021. Epub Nov 06, 2020. https://doi.org/10.1590/1982-4327e3032.

Straus, M. A., \& Gozjolko, K. L. (2014). "Intimate terrorism" and gender differences in injury of dating partners bymale and female university students. Journal of FamilyViolence, 29(1), 5165. doi:10.1007/s10896-013-9560-7

Johnson, M. P. (2011). Gender and types of intimate partner violence: A response to an antifeminist literature review. Aggression and Violent Behavior, 16(4), 289-296. doi:10.1016/j.avb.2011.04.006.

Barros, S.C. Homicídios e violência prévia contra a mulher em Pernambuco. Revista Baiana de Saúde Pública. v. 42, n. 3, p. 570-572jul./set. 2018 